\title{
Fair Scheduling with Deadline Guarantees in Single-hop Networks
}

\author{
Hussam Ahmed, Krishna Jagannathan, Srikrishna Bhashyam \\ Department of Electrical Engineering \\ IIT Madras, Chennai, India
}

\begin{abstract}
We address the problem of simultaneously ensuring long-term fairness and deterministic delay guarantees for realtime traffic over a single-hop network. Specifically, we propose a network control policy that maximises a concave utility function of the average throughput of each flow, while guaranteeing that each packet is delivered within a deterministic deadline. Although this problem has been addressed in the past, prior work makes restrictive assumptions, by allowing only binary packet arrival and service processes at each link. The present paper allows for any bounded burst size distributions for the arrival and service processes.
\end{abstract}

\section{INTRODUCTION}

In this paper, we tackle the problem of scheduling for network utility maximisation in a generic single hop network, with deterministic delay constraints for every packet. In particular, each packet has to reach its destination by a certain deadline. Packets that do not reach their destination by the deadline are useless, and are dropped. Our work is motivated by the significant growth of real-time traffic, which demand both stringent delay constraints for each packet, as well as a satisfactory long-term rate.

We develop a scheduling mechanism that maximises a concave utility function of the long term rates, while ensuring that the packets that are scheduled do reach their destination before the deadline. The delay bound is guaranteed by tracking the waiting time of the head-of-line data in all the queues in every slot and by making the scheduling decisions based on those waiting times. Thus, our policy ensures both long-term fairness for competing flows, and the deadline constraints for each packet.

The scheduling problem in wireless networks has been well studied for over two decades. Much of the existing literature in this area is based on the Lyapunov drift technique for provably stable scheduling of a queuing system, which was introduced in [1], [2]. Since then, these Lyapunov methods, which explicitly take queue lengths into account for making scheduling decisions, have been applied in various contexts including high-speed switches [3], satellites [4], wireless [5], and optical networks [6]. In all these cases, the goal is to guarantee stability of the queuing system, whenever the traffic is within the stability region.

In cases when the traffic arrival rate to a network may lie outside the stability region, Lyapunov methods have been used to obtain joint congestion control and scheduling policies, so as to maximise a concave utility function of the long term throughputs obtained by each flow [7]-[9]. These papers also propose queue length based allocation rules, and expected delay bounds are obtained via corresponding bounds on the queue lengths, since the two quantities are simply related through Little's law [10].

Although expected delay bounds for queue length based policies have been known for a while, the increasing importance of real-time traffic has engendered recent interest in providing more stringent delay guarantees for every delivered packet. An early work which addressed deadline constrained scheduling for multiple real-time streams sharing a wireless channel is [11]. More recently, in [12], the authors address the scheduling problem in the presence of elastic and inelastic traffic, and maximise the utility of the elastic traffic while ensuring packet delay deadlines for the inelastic traffic. This was extended to incorporate heterogenous delay constraints in [13]. Scheduling real-time flows over multi-hop networks was studied in [14]. Also notable are the papers [15], [16], where the authors use Blackwell's approachability theory to guarantee certain delay guarantees and delivery ratios for realtime traffic.

Our present paper is most directly related to [17], where the author proposes a delay-based Lyapunov technique to maximise a network utility function of the long-term throughput, while ensuring that each delivered packet meets a deterministic deadline constraint. Incidentally, head-of-line delay-based scheduling was first introduced in the switching literature [3], [18]. Scheduling based on the head-of-line delay, as opposed to the queue backlog, has the advantage of giving a more direct control over the delay. In particular, [17] guarantees a deterministic delay guarantee by tracking the waiting time of the head-of-line packets in all the queues in every slot, and making the scheduling decisions based on those waiting times.

However, the results in [17] hold only under restrictive assumptions on the arrival and service processes at each link - in particular, when the arrivals are Bernoulli distributed, and the service process is binary in each time slot. In this paper, we adopt the framework of concavely extended utility function from [17], and generalise the results to allow for any bounded arrival process and service rate at each link. As it turns out, the congestion control, packet drop, and link scheduling decisions decouple under the Bernoulli arrivals and binary service assumptions made in [17]. However, in the more general setting of the present paper, the problem is considerably more complex, and the optimal packet drop decisions become coupled with the scheduling decisions. 
Further, the general arrival and service processes necessitate more elaborate bounding of the Lyapunov drift, which we accomplish by identifying a stopping rule, and invoking Wald's equality and a second moment bound. In sum, we solve the network utility maximisation problem subject to a deadline constraint on each delivered packet, under fairly general arrival and service processes at each link

The remainder of this paper is organised as follows. Section II describes the network model and Section III defines the optimisation problem. Section IV derives bounds on the Lyapunov drift for general arrival and service processes. The optimal control policy is proposed in Section $\mathrm{V}$, and its optimality properties are proven. Section VI concludes the paper.

\section{NETWORK MODEL}

Consider a time slotted single-hop network with $K$ links numbered as $\{1,2, \ldots, K\}$. Data packets arrive randomly every slot and are put into separate queues for different links. Let $A_{i}(t)$ be the amount of data arriving at queue $i$ during slot $t$. The arrivals to the network are assumed to be i.i.d. over slots and independent over different queues. We assume that the amount of data that arrives to each queue in one slot is deterministically bounded above, i.e., $A_{i}(t) \leq A_{\max }$ for all $i$ and $t$. The special case of at most one packet arrival to each link per slot was considered in [17]. Let $\underline{\lambda}=\left(\lambda_{1}, \lambda_{2}, \ldots \lambda_{K}\right)$ be the arrival rate vector, where $\lambda_{i}=E\left\{A_{i}(t)\right\}$. Let $Q(t)=\left(Q_{1}(t), Q_{2}(t), \ldots, Q_{K}(t)\right)$ be the queue-length vector for the network at the beginning of slot $t$. The packets in the queue are marked with their integer arrival times which are then used to determine the waiting time in the system. The queue evolution is given by the following equation.

$$
Q_{i}(t+1)=\max \left\{Q_{i}(t)-\mu_{i}(t)-D_{i}(t), 0\right\}+A_{i}(t),
$$

where $\mu_{i}(t)$ is the service given to queue $i$ during slot $t$ and $D_{i}(t)$ is the amount of data dropped from the $i^{t h}$ queue during slot $t$. The link capacities are assumed to be time varying and are denoted by $\underline{C}(t)=\left(C_{1}(t), C_{2}(t), \ldots C_{K}(t)\right)$ for the $K$ links during slot $t$. We assume that the capacity of the link is upper bounded by $C_{\max }$. In [17], each link can transmit at most one packet per slot. Let $\underline{x}(t)=\left(x_{1}(t), x_{2}(t), \ldots x_{K}(t)\right)$ be the transmission rate vector during slot $t$. The service rate $\mu_{i}(t)$ is given as follows.

$$
\mu_{i}(t)=x_{i}(t) 1_{i}(t),
$$

where $1_{i}(t)$ is an indicator variable that takes 1 when the transmission through link $i$ is successful. At each $t$, we assume that $1_{i}(t)$ is known to the controller for each $i$.

For each queue $i$, define $Y_{i}(t)=\lambda_{i}-D_{i}(t)$. Let $y$ be the time average expectation of $Y_{i}(t)$

$$
\underline{y}=\underline{\lambda}-\lim _{t \rightarrow \infty} \frac{1}{t} \sum_{\tau=0}^{t} \mathbb{E}\{\underline{D}(\tau)\} .
$$

The vector $y$ is the difference between the rate of arrivals and the rate of packet drops, and hence it represents the throughtput vector provided the queues are stable.

\section{THE OPTIMISATION OBJECTIVE}

As in [17], the goal is to propose a delay-based transmission scheme with data dropping that the solves the following problem:

$$
\max _{\underline{y}} g(\underline{y})
$$

$$
\begin{aligned}
& \text { subject to: } \quad \underline{y} \in \Lambda \text {, } \\
& 0 \leq y_{i} \leq \lambda_{i}, \forall i \text {, }
\end{aligned}
$$

where $g(y)$ is a continuous and concave utility function of the $K$-dimensional vector $y$, and $\Lambda$ is the set of all long-term throughput vectors that the system can support. The function is assumed to be defined over the hyper-cube $\mathcal{Y}=\{y \mid 0 \leq$ $\left.y_{i} \leq A_{\max } \forall i\right\}$. We make the following additional assumption as in [17].

Assumption 1 : For each queue $i$, for any vectors $\underline{y}$ and $\underline{w}$ such that $\underline{y} \in \mathcal{Y}, \underline{w} \in \mathcal{Y}$ and $\underline{y}+\underline{w} \in \mathcal{Y}$ we have :

$$
g(\underline{y}+\underline{w}) \leq g(\underline{y})+\sum_{i=1}^{K} \nu_{i} w_{i}
$$

where $\nu_{i} \geq 0 \forall i$. Note that (2) is true if, for each $i$, the $i^{\text {th }}$ right partial derivative of $g()$ is upper bounded by a finite constant $\nu_{i}$.

Let $g^{*}$ be the maximum value of the objective in the problem (1). Our aim is to find a policy which achieves a utility close to $g^{*}$ and simultaneously guarantees a deterministic delay bound for the non dropped data.

\section{A. Concave Extension of Utility Function}

Following [17], we define a concave extension of the utility function, which plays an important role in bounding the delay. Supposing that $g(\underline{y})$ satisfies Assumption 1, we define $\hat{g}(\underline{y})$ over the extended hyper-cube $\hat{\mathcal{Y}}=\left\{\underline{y} \mid-A_{\max } \leq y_{i} \leq\right.$ $A_{\max } \forall i$ \} as a concave extension of $g(\underline{y})$ given by,

$$
\hat{g}(\underline{y})=g(\max \{\underline{y}, 0\})+\sum_{i=1}^{K} \nu_{i} \min \left\{y_{i}, 0\right\},
$$

where $\max \{\underline{y}, 0\}=\left(\max \left\{y_{1}, 0\right\}, \ldots, \max \left\{y_{K}, 0\right\}\right)$. Since (2) holds, we have the following inequality.

$$
\hat{g}(\underline{y}) \leq \hat{g}\left(\underline{y}_{i}\right)+\nu_{i}\left(y_{i}+A_{\max }\right),
$$

where $\underline{y}_{i}$ is formed from $\underline{y}$ by replacing $y_{i}$ by $-A_{\max }$. 


\section{B. Equivalent Problem with Virtual Queues}

The optimisation problem (1) can be easily transformed to the following problem using an auxillary vector $\phi(t)$.

$$
\max _{\underline{\phi}, \underline{y}} \hat{g}(\underline{\phi})
$$

subject to:

$$
\begin{gathered}
y_{i} \geq \phi_{i} \forall i, \\
-A_{\max } \leq \phi_{i} \leq A_{\max }, \quad \forall i, \\
\underline{\bar{Q}}<\infty,
\end{gathered}
$$

where $\underline{Q}$ and $\underline{y}$ are achievable on the network and $\underline{Q}=$ $\limsup _{t \rightarrow \infty} \frac{1}{t} \sum_{\tau=0}^{t} \mathbb{E}\{\underline{Q}(\tau)\}$.

We solve the above problem by using the Lyapunov optimisation technique for stabilising a set of virtual queues $Z(t)=\left(Z_{1}(t), Z_{2}(t), \ldots, Z_{K}(t)\right)$ with the update equation as follows. For the $i^{\text {th }}$ queue,

$$
Z_{i}(t+1)=\max \left\{Z_{i}(t)-\lambda_{i}+D_{i}(t)+\phi_{i}(t), 0\right\} .
$$

Stabilising this virtual queues ensures that the first constraint in problem (4) is satisfied. Note that the virtual queue update equation needs the knowledge of the actual arrival rate vector $\underline{\lambda}$. In order to incorporate the delay into the resource allocation policy, we define $W_{i}(t)$ as the waiting time of the head-ofline data in the $i^{t h}$ queue on slot $t$. Let $W_{i}(t)=0$ if the queue $i$ is empty. Let $N_{i}(t)$ be the amount of head-of-line data in the $i^{\text {th }}$ queue. Define $1_{Q_{i}}(t)$ as an indicator variable that is 1 if $Q_{i}(t)>0$, and is zero if the queue is empty. Define $1_{D_{i}}(t)$ as an indicator random variable which takes a value 1 whenever $D_{i}(t)>0$ and zero otherwise. Let $1_{A_{i}(t)}$ be an indicator variable which takes 1 if $A_{i}(t)>0$ and zero otherwise. Let $1_{N_{i}}(t)$ be an indicator variable which takes a value 1 if $N_{i}(t) \geq \mu_{i}(t)$ and zero otherwise. We observe that $W_{i}(t)$ satisfies the following update equation.

$$
\begin{aligned}
& W_{i}(t+1)=\left(1-1_{Q_{i}}(t)\right) 1_{A_{i}(t)} \\
& \quad+1_{Q_{i}}(t) \max \left\{W_{i}(t)+1-\rho_{i}(t), 0\right\},
\end{aligned}
$$

where $\rho_{i}(t)$ represents the time after which the data at the head-of-line in the $i^{t h}$ queue at the end of slot $t$ (after the service during slot $t$ ) have arrived with respect to the arrival epoch of the head-of-line data in the beginning of the slot. We can express $\rho_{i}(t)$ as follows.

$$
\begin{aligned}
\rho_{i}(t) & = \begin{cases}0, & 1_{D_{i}}(t)=0 \& 1_{N_{i}}(t)=1 \\
T_{i}(t), & 1_{D_{i}}(t)=1 \& 1_{N_{i}}(t)=1, \\
J_{i}(t), & 1_{N_{i}}(t)=0\end{cases} \\
& =\left(1-1_{N_{i}}(t)\right) J_{i}(t)+1_{N_{i}}(t) 1_{D_{i}}(t) T_{i}(t)
\end{aligned}
$$

where

$$
T_{i}(t)=\min \left\{\omega \mid A_{i}\left(t-W_{i}(t)+\omega\right)>0, \omega>0\right\},
$$

and

$$
J_{i}(t)=\min \left\{\omega \mid \sum_{\tau=1}^{\omega} A_{i}\left(t-W_{i}(t)+\tau\right)>\mu_{i}(t)-N_{i}(t)\right\} .
$$

The update equation of the waiting time of the head-of-line data given by (6) can be understood as follows: If the queue is empty, the value of $W_{i}(t+1)$ is 1 if and only if there is a new arrival in the current slot $t$. Alternatively if the queue is non empty, $\mu_{i}(t)$ amount of data is served from the queue. Now if $\mu_{i}(t)$ is less than the amount of head-of-line data in the queue (i.e., $1_{N_{i}}(t)=1$ ), then the remaining packets are either dropped or retained in the queue. If the remaining packets are not dropped, then $\rho_{i}(t)=0$ in (7). Hence $W_{i}(t+1)$ is $W_{i}(t)+1$ in this case. If the remaining packets are dropped, then $W_{i}(t+1)$ becomes $W_{i}(t)+1-T_{i}(t)$, i.e., the packet which arrived $T_{i}(t)$ slots after the current head-of-line paket will become the new head-of-line packet in the next slot. On the other hand, if $1_{N_{i}}(t)=0$, i.e., the number of serviced packets is larger than the number of head-of-line packets, the data which arrived $J_{i}(t)$ slots after the current head-of-line data will become the new head-of-line in the next slot which makes $W_{i}(t+1)$ to be $W_{i}(t)+1-J_{i}(t)$.

Without loss of generality, assume that $\lambda_{i}>0$ for all the queues. We obtain our delay-based scheduling policy using the Lyapunov optimisation framework in [7].

$\begin{array}{ccccc}\text { Define } & \Theta(t) & = & {[\underline{Z}(t) ; \underline{W}(t) ; \underline{N}(t) ; I(t)],} \\ \text { where } & W(t) & = & \left(W_{1}(t), W_{2}(t), \ldots W_{K}(t)\right),\end{array}$ $\underline{N}(t)=\left(N_{1}(t), N_{2}(t), \ldots N_{K}(t)\right), \quad$ and $I(t)=$ $\left(1_{1}(t), 1_{2}(t), \ldots 1_{K}(t)\right)$. We consider the following nonnegative Lyapunov function:

$$
L(\Theta(t))=\frac{1}{2} \sum_{i=1}^{K} Z_{i}(t)^{2}+\frac{1}{2} \sum_{i=1}^{K} k_{i} W_{i}(t)^{2},
$$

where $k_{i}=1-\mathbb{P}\left\{A_{i}(t)=0\right\}$. The conditional Lyapunov drift $\Delta(\Theta(t))$ is defined as:

$$
\Delta(\Theta(t))=\mathbb{E}\{L(\Theta(t+1))-L(\Theta(t)) \mid \Theta(t)\} .
$$

\section{BOUNDING THE DRIFT-MINUS-UTILITY}

The drift-minus-utility expression is given by

$$
\zeta=\Delta(\Theta(t))-V \mathbb{E}\{\hat{g}(\underline{\phi}(t)) \mid \Theta(t)\},
$$

where $V$ is a non-negative control parameter. We first obtain a bound on the drift-minus-utility and then minimise the bound to arrive at the policy.

First, we show that $\mathbb{E}\left\{J_{i}(t)^{2} \mid \Theta(t)\right\}$ is finite in the following lemma.

Lemma 1. In every slot $t$, for any queue $i$ :

$$
\mathbb{E}\left\{J_{i}(t)^{2} \mid \Theta(t)\right\}<\infty .
$$

Proof: Every slot $t$, conditioning on the service $\mu_{i}(t)$, the random variable $J_{i}(t)$ can be viewed as a stopping rule which depends on i.i.d. arrivals into the queue $i$. Now we use [10, Lemma 1, Section 7.5] which asserts that $J_{i}(t)$ has all its conditional moments to be finite. Hence we have,

$$
\mathbb{E}\left\{J_{i}(t)^{2} \mid \Theta(t), \mu_{i}(t)\right\}<\infty .
$$

Now by taking the expectation over $\mu_{i}(t)$, the lemma follows. 

$J_{i}(t)$.

Further, we have the following lemma relating $\rho_{i}(t)$ and

Lemma 2. In every slot $t$, for any queue $i$ :

$$
\rho_{i}(t) \geq J_{i}(t)-1 \text {. }
$$

Proof: Note that $J_{i}(t)$ becomes 1 whenever $1_{N_{i}}(t)=1$. Hence, whenever $1_{N_{i}}(t)=1$, irrespective of whether data are dropped or not, $\rho_{i}(t) \geq J_{i}(t)-1=0$. Now if $1_{N_{i}}(t)=0$, then $\rho_{i}(t)=J_{i}(t) \geq J_{i}(t)-1$.

Now, we obtain bounds on the drift and drift-minus-utility.

Lemma 3. In every slot $t$, the Lyapunov drift satisfies:

$$
\begin{aligned}
\Delta(\Theta(t)) \leq & B_{1}+\sum_{i=1}^{K} k_{i} W_{i}(t)+\sum_{i=1}^{K} \frac{k_{i}\left(1-1_{N_{i}}(t)\right) W_{i}(t) N_{i}(t)}{\lambda_{i}} \\
& -\sum_{i=1}^{K} Z_{i}(t) \mathbb{E}\left\{Y_{i}(t)-\phi_{i}(t) \mid \Theta(t)\right\} \\
& -\sum_{i=1}^{K} \frac{k_{i}\left(1-1_{N_{i}}(t)\right) W_{i}(t) \mathbb{E}\left\{\mu_{i}(t) \mid \Theta(t)\right\}}{\lambda_{i}} \\
& -\sum_{i=1}^{K} 1_{N_{i}}(t) W_{i}(t) \mathbb{E}\left\{1_{D_{i}}(t) \mid \Theta(t)\right\} .
\end{aligned}
$$

where $B_{1}$ is a finite positive constant.

Proof: We get the following inequality by squaring (5).

$$
\begin{aligned}
Z_{i}(t+1)^{2} \leq Z_{i}(t)^{2} & +\phi_{i}(t)^{2}+Y_{i}(t)^{2} \\
& -2 Z_{i}(t)\left(Y_{i}(t)-\phi_{i}(t)\right)-2 Y_{i}(t) \phi_{i}(t)
\end{aligned}
$$

Since the arrivals in any slot are bounded, the term $Y_{i}(t)^{2}+$ $\phi_{i}(t)^{2}-2 Y_{i}(t) \phi_{i}(t)$ is bounded. Rearranging, summing over all the queues, and taking conditional expection, we get

$$
\begin{aligned}
& \mathbb{E}\left\{\sum_{i=1}^{K}\left(Z_{i}(t+1)^{2}-Z_{i}(t)^{2}\right) \mid \Theta(t)\right\} \\
& \quad \leq B_{1}^{\prime}-2 \sum_{i=1}^{K} \mathbb{E}\left\{Z_{i}(t)\left(Y_{i}(t)-\phi_{i}(t)\right) \mid \Theta(t)\right\},
\end{aligned}
$$

where $B_{1}^{\prime}$ is a finite positive constant. Now squaring (6), we get the following bound.

$$
\begin{aligned}
& W_{i}(t+1)^{2} \leq 1+\left(W_{i}(t)+1-\rho_{i}(t)\right)^{2} \\
& =1+W_{i}(t)^{2}+\left(1-\rho_{i}(t)\right)^{2}+2 W_{i}(t)\left(1-\rho_{i}(t)\right)
\end{aligned}
$$

Using Lemmas 1 and 2, we can bound $\mathbb{E}\left\{\left(1-\rho_{i}(t)\right)^{2} \mid \Theta(t)\right\}$ by a finite positive constant. Rearranging, summing over all the queues, taking conditional expectation, and using Lemma 1, we get

$$
\begin{aligned}
\mathbb{E}\left\{\sum_{i=1}^{K}\right. & \left.k_{i}\left(W_{i}(t+1)^{2}-W_{i}(t)^{2}\right) \mid \Theta(t)\right\} \\
& \leq \hat{B}_{1}+2 \sum_{i=1}^{K} k_{i} \mathbb{E}\left\{W_{i}(t)\left(1-\rho_{i}(t)\right) \mid \Theta(t)\right\},
\end{aligned}
$$

where $\hat{B}_{1}$ is a finite positive constant. Using

$$
\begin{aligned}
\mathbb{E}\left\{\rho_{i}(t) \mid \Theta(t)\right\} & =\left(1-1_{N_{i}}(t)\right) \mathbb{E}\left\{J_{i}(t) \mid \Theta(t)\right\} \\
& +1_{N_{i}}(t) \mathbb{E}\left\{1_{D_{i}}(t) \mid \Theta(t)\right\} \mathbb{E}\left\{T_{i}(t) \mid \Theta(t)\right\}
\end{aligned}
$$

we get

$$
\begin{aligned}
& \mathbb{E}\left\{\sum_{i=1}^{K} k_{i}\left(W_{i}(t+1)^{2}-W_{i}(t)^{2}\right) \mid \Theta(t)\right\} \\
\leq & \hat{B}_{1}+2 \sum_{i=1}^{K} k_{i} W_{i}(t)-2 \sum_{i=1}^{K} k_{i}\left(1-1_{N_{i}}(t)\right) W_{i}(t) \mathbb{E}\left\{J_{i}(t) \mid \Theta(t)\right\} \\
& -2 \sum_{i=1}^{K} k_{i} 1_{N_{i}}(t) \mathbb{E}\left\{1_{D_{i}}(t) \mid \Theta(t)\right\} W_{i}(t) \mathbb{E}\left\{T_{i}(t) \mid \Theta(t)\right\} .
\end{aligned}
$$

In writing (13), we have used the fact that our decision to drop $1_{D_{i}}(t)$ is independent of the inter-arrival time $T_{i}(t)$.

Next, from equations (12) and (14), we get

$$
\begin{aligned}
\Delta(\Theta(t)) \leq & B_{1}+\sum_{i=1}^{K} k_{i} W_{i}(t)-\sum_{i=1}^{K} Z_{i}(t) \mathbb{E}\left\{Y_{i}(t)-\phi_{i}(t) \mid \Theta(t)\right\} \\
& -\sum_{i=1}^{K} k_{i}\left(1-1_{N_{i}}(t)\right) W_{i}(t) \mathbb{E}\left\{J_{i}(t) \mid \Theta(t)\right\} \\
& -\sum_{i=1}^{K} k_{i} 1_{N_{i}}(t) W_{i}(t) \mathbb{E}\left\{1_{D_{i}}(t) \mid \Theta(t)\right\} \mathbb{E}\left\{T_{i}(t) \mid \Theta(t)\right\} .
\end{aligned}
$$

In every slot $t$, conditioned on the service $\mu_{i}(t)$, the random variable $J_{i}(t)$ can be viewed as a stopping rule which depends on the set of independent arrivals to the $i^{\text {th }}$ queue. Applying Wald's equality [10], we get

$$
\mathbb{E}\left\{A^{J_{i}(t)} \mid \mu_{i}(t), \Theta(t)\right\}=\lambda_{i} \mathbb{E}\left\{J_{i}(t) \mid \mu_{i}(t), \Theta(t)\right\} .
$$

where $A^{J_{i}(t)}=\sum_{\tau=1}^{J_{i}(t)} A_{i}\left(t-W_{i}(t)+\tau\right)$. Clearly, by definition of $J_{i}(t), A^{J_{i}(t)}>\mu_{i}(t)-N_{i}(t)$. Thus,

$$
\mathbb{E}\left\{J_{i}(t) \mid \mu_{i}(t), \Theta(t)\right\}>\frac{\mu_{i}(t)-N_{i}(t)}{\lambda_{i}} .
$$

Now taking expectation over $\mu_{i}(t)$ we get,

$$
\mathbb{E}\left\{J_{i}(t) \mid \Theta(t)\right\}>\frac{\mathbb{E}\left\{\mu_{i}(t) \mid \Theta(t)\right\}-N_{i}(t)}{\lambda_{i}} .
$$

Finally, note that $T_{i}(t)$ which is an inter-arrival time, is geometrically distributed with success parameter $k_{i}$, so that

$$
\mathbb{E}\left[T_{i}(t) \mid \Theta(t)\right]=1 / k_{i} .
$$

Using (18) and (19) in (15), the lemma follows. 
Lemma 4. In every slot $t$, the drift-minus-utility satisfies:

$$
\begin{aligned}
\zeta \leq & B_{1}+\sum_{i=1}^{K} k_{i} W_{i}(t)+\sum_{i=1}^{K} \frac{k_{i}\left(1-1_{N_{i}}(t)\right) W_{i}(t) N_{i}(t)}{\lambda_{i}} \\
& -\mathbb{E}\left\{V \hat{g}(\underline{\phi}(t))-\sum_{i=1}^{K} Z_{i}(t) \phi_{i}(t) \mid \Theta(t)\right\} \\
& -\sum_{i=1}^{K} Z_{i}(t) \mathbb{E}\left\{Y_{i}(t) \mid \Theta(t)\right\} \\
& -\sum_{i=1}^{K} \frac{k_{i}\left(1-1_{N_{i}}(t)\right) W_{i}(t) \mathbb{E}\left\{\mu_{i}(t) \mid \Theta(t)\right\}}{\lambda_{i}} \\
& -\sum_{i=1}^{K} 1_{N_{i}}(t) W_{i}(t) \mathbb{E}\left\{1_{D_{i}}(t) \mid \Theta(t)\right\},
\end{aligned}
$$

where $B_{2}$ is a finite constant.

Proof: Using the bound for drift in Lemma 3 in (10), we obtain the bound for drift-minus-utility.

In the next section, we present our delay based scheduling policy that chooses the optimal $\underline{x}(t), \underline{\phi}(t)$, and $\underline{D}(t)$.

\section{Proposed Resource Allocation Policy}

We propose a control policy that minimises the upper bound in Lemma 4. Since the first three positive terms cannot be controlled, our policy essentially minimises the negative terms, which is equivalent to maximising

$$
\begin{gathered}
\mathbb{E}\left\{V \hat{g}(\underline{\phi}(t))-\sum_{i=1}^{K} Z_{i}(t) \phi_{i}(t) \mid \Theta(t)\right\}+\sum_{i=1}^{K} Z_{i}(t) \mathbb{E}\left\{Y_{i}(t) \mid \Theta(t)\right\} \\
+\sum_{i=1}^{K} \frac{k_{i}\left(1-1_{N_{i}}(t)\right) W_{i}(t) \mathbb{E}\left\{\mu_{i}(t) \mid \Theta(t)\right\}}{\lambda_{i}} \\
+\sum_{i=1}^{K} 1_{N_{i}}(t) W_{i}(t) \mathbb{E}\left\{1_{D_{i}}(t) \mid \Theta(t)\right\}
\end{gathered}
$$

During slot $t$, the controller observes $\Theta(t)$, and implements the following policy.

1) Congestion control step:

Choose $\underline{\phi}(t)$ as the solution to the following problem:

$$
\max V \hat{g}(\underline{\phi}(t))-\sum_{i=1}^{K} Z_{i}(t) \phi_{i}(t)
$$

subject to $-A_{\max } \leq \phi_{i}(t) \leq A_{\max }, \forall i$.

2) Transmission rate allocation:

Choose $\underline{x}^{*}(t)$ as in (23), where $1_{N_{i}}(t)=1$ if $N_{i}(t)>$ $x_{i}(t) 1_{i}(t)$, and 0 otherwise, and $1_{D_{i}}(t)=1$ if $1_{N_{i}}(t)=$ 1 and $W_{i}(t)>Z_{i}(t)\left(N_{i}(t)-x_{i}(t) 1_{i}(t)\right)$, and 0 otherwise.

3) Dropping decision:

If $N_{i}(t)>x_{i}^{*}(t) 1_{i}(t)$ and $W_{i}(t)>$ $Z_{i}(t)\left(N_{i}(t)-x_{i}^{*}(t) 1_{i}(t)\right)$, drop the head-of-line packets at link $i$, for each $i=1, \ldots, K$.

\section{A. Minimising the drift-minus-utility bound}

Theorem 1. The delay based resource allocation policy given above maximises the expression in (20).

Proof: Notice that $\phi_{i}(t)$ appears only in the first expectation term in (20). Therefore, they can be optimally chosen by maximising the following quantity for a given $\Theta(t)$ :

$$
V \hat{g}(\underline{\phi}(t))-\sum_{i=1}^{K} Z_{i}(t) \phi_{i}(t)
$$

subject to $-A_{\max } \leq \phi_{i}(t) \leq A_{\max }$ for all queues $i$. This gives the congestion control step of the policy.

Next, we have to maximise the following quantity by optimally choosing the transmission vector $\underline{x}(t)$ and the packet drop vector $\underline{D}(t)$ :

$$
\begin{aligned}
&-\sum_{i=1}^{K} Z_{i}(t) \mathbb{E}\left\{D_{i}(t) \mid \Theta(t)\right\} \\
&+\sum_{i=1}^{K} \frac{k_{i}\left(1-1_{N_{i}}(t)\right) W_{i}(t) \mathbb{E}\left\{\mu_{i}(t) \mid \Theta(t)\right\}}{\lambda_{i}} \\
& \\
& \quad+\sum_{i=1}^{K} 1_{N_{i}}(t) W_{i}(t) \mathbb{E}\left\{1_{D_{i}}(t) \mid \Theta(t)\right\} .
\end{aligned}
$$

In writing the above, we have used $Y_{i}(t)=\lambda_{i}-D_{i}(t)$.

Now, let us fix some $\underline{x}(t)$, and decide the corresponding $\underline{D}(t)$. Since the channel state at slot $t$ is known, $\mu_{i}(t)=$ $x_{i}(t) 1_{i}(t)$ is also known for this fixed $\underline{x}(t)$. This allows us to calculate $1_{N_{i}}(t)$ also. Observe next that when $1_{N_{i}}(t)=0$, (i.e., when $\mu_{i}(t) \geq N_{i}(t)$,), only the first two tems in (22) are non-zero. Thus, $D_{i}(t)=0$ is optimal. In other words, if all head-of-line packets can be served during the slot, it is optimal to not drop any packets.

Next, when $1_{N_{i}}(t)=1$, the second term in (22) is zero. Therefore, we need to maximise

$$
\sum_{i=1}^{K} W_{i}(t) \mathbb{E}\left\{1_{D_{i}}(t) \mid \Theta(t)\right\}-Z_{i}(t) \mathbb{E}\left\{D_{i}(t) \mid \Theta(t)\right\} .
$$

If we decide not to drop for some $i$, we see that the corresponding term in the above expression is zero. Thus, it makes sense to drop the remaining $N_{i}(t)-\mu_{i}(t)$ headof-line packets, only if $W_{i}(t)>Z_{i}(t)\left(N_{i}(t)-\mu_{i}(t)\right)$. To summarise, for a fixed $\underline{x}(t)$ and each $i=1, \ldots, K$, we drop all $N_{i}(t)-\mu_{i}(t)$ head-of-line packets, if and only if $1_{N_{i}}(t)=1$ and $W_{i}(t)>Z_{i}(t)\left(N_{i}(t)-\mu_{i}(t)\right)$. This determines the optimal drop policy for a given $\underline{x}(t)$.

The optimal $\underline{x}^{*}(t)$ is now found by back-substituting the drop policy in (22), and maximising over all feasible $\underline{x}(t)$. That is,

$$
\begin{aligned}
& \underline{x}^{*}(t)=\underset{\underline{x}(t)}{\arg \max _{i=1}} \sum_{N_{i}}^{K}\left[\frac{k_{i}}{\lambda_{i}}\left(1-1_{N_{i}}(t)\right) W_{i}(t) x_{i}(t) 1_{i}(t)\right. \\
& \left.+1_{N_{i}}(t) W_{i}(t) 1_{D_{i}}(t)-Z_{i}(t) 1_{D_{i}}(t)\left(N_{i}(t)-x_{i}(t) 1_{i}(t)\right)\right] .
\end{aligned}
$$


Finally, the overall optimal drop decision $\underline{D}^{*}(t)$ is the optimal drop decision corresponding to $\underline{x}^{*}(t)$.

At this point, we pause briefly to compare with the drop decision in [17], where due to i.i.d. Bernoulli arrivals and ON/OFF channels, $\mathbb{E}\left\{1_{D_{i}}(t) \mid \Theta(t)\right\}=\mathbb{E}\left\{D_{i}(t) \mid \Theta(t)\right\}$. Therefore the drop decision in [17] is based on comparing $W_{i}(t)$ with $Z_{i}(t)$, which is what our policy simplifies to, for the special case of Bernoulli arrivals and ON/OFF channels.

\section{B. Delay bound}

The following lemma is useful to prove that the delay is deterministically bounded.

Lemma 5. If $Z_{i}(t)>V \nu_{i}$ for a particular slot and queue $i$, then the congestion control step in the above policy chooses $\phi_{i}(t)=-A_{\max }$ for that slot.

Proof: The proof is similar to [17, Lemma 3]. By (3), we know that for any vector $\underline{\phi}(t)$ such that $-A_{\max } \leq \phi_{i}(t) \leq$ $A_{\max }$,

$$
\begin{aligned}
& V \hat{g}(\underline{\phi}(t))-\sum_{m=1}^{K} Z_{m}(t) \phi_{m}(t) \\
& \leq V \hat{g}\left(\underline{\phi}_{i}(t)\right)+V \nu_{i}\left(\phi_{i}(t)+A_{\max }\right)-\sum_{m=1}^{K} Z_{m}(t) \phi_{m}(t)
\end{aligned}
$$

Because $V \nu_{i}<Z_{i}(t)$, the upper bound is maximised at $\phi_{i}(t)=-A_{\max }$ and equality holds if and only if $\phi_{i}(t)=$ $-A_{\max }$.

Theorem 2. The proposed resource allocation policy ensures a deterministic bound on both the virtual queue size and the delay of the head-of-line data in every queue in every slot. More precisely, for any queue $i$,

$Z_{i}(t) \leq\left\lceil V \nu_{i}\right\rceil+2 A_{\max }$ and $W_{i}(t) \leq A_{\max }\left(\left\lceil V \nu_{i}\right\rceil+2 A_{\max }\right)$.

Proof: Consider the $i^{\text {th }}$ queue. At $t=0$, when the queues are empty, clearly, $Z_{i}(0) \leq\left\lceil V \nu_{i}\right\rceil+2 A_{\max }$ and $W_{i}(0) \leq$ $A_{\max }\left(\left\lceil V \nu_{i}\right\rceil+2 A_{\max }\right)$. Hence the theorem holds for $t=0$. We use this as the basis for the following proof by induction. We assume that (24) holds for given $t$.

From (5) we have,

$$
Z_{i}(t+1) \leq Z_{i}(t)+D_{i}(t)+\phi_{i}(t) \leq Z_{i}(t)+2 A_{\max } .
$$

If $Z_{i}(t) \leq\left\lceil V \nu_{i}\right\rceil$, then $Z_{i}(t+1) \leq\left\lceil V \nu_{i}\right\rceil+2 A_{\max }$. Now if $Z_{i}(t)>\left\lceil V \nu_{i}\right\rceil, \phi_{i}(t)=-A_{\max }\left(\right.$ by Lemma 5), $D_{i}(t) \leq$ $A_{\max }$, and hence $Z_{i}(t+1) \leq Z_{i}(t) \leq\left\lceil V \nu_{i}\right\rceil+2 A_{\max }$. Therefore, by induction, $Z_{i}(t)$ is bounded above as in (24).

Similarly, from (6), we have

$$
W_{i}(t+1) \leq W_{i}(t)+1 \text {. }
$$

If $W_{i}(t) \leq A_{\max }\left(\left\lceil V \nu_{i}\right\rceil+2 A_{\max }\right)-1$, then $W_{i}(t+1) \leq$ $A_{\max }\left(\left\lceil V \nu_{i}\right\rceil+2 A_{\max }\right)$. Now if $W_{i}(t)>A_{\max }\left(\left\lceil V \nu_{i}\right\rceil+\right.$ $\left.2 A_{\max }\right)-1$, then $W_{i}(t)=A_{\max }\left(\left\lceil V \nu_{i}\right\rceil+2 A_{\max }\right)$, which makes $W_{i}(t) \geq Z_{i}(t) A_{\max } \geq Z_{i}(t)\left(N_{i}(t)-x_{i}(t) 1_{i}(t)\right)$. Therefore, the head-of-line data will be completely drained off (either by successful transmission or by dropping). Therefore, $W_{i}(t+1) \leq W_{i}(t) \leq A_{\max }\left(\left\lceil V \nu_{i}\right\rceil+2 A_{\max }\right)$. By induction, the theorem holds for all $t$.

\section{Utility gap}

Theorem 3. The proposed resource allocation policy ensures the delay of all non-dropped data to be less than or equal to $D$ slots, with total throughput-utility that differs

by $\mathcal{O}(1 / D)$.

Proof: Theorem 2 shows that the delay of any successfully transmitted packet in the $i^{\text {th }}$ queue is bounded by $A_{\max }\left(\left\lceil V \nu_{i}\right\rceil+2 A_{\max }\right)$ slots. Hence, by choosing $V$ such that $D=A_{\max }\left(\left\lceil V \nu_{\max }\right\rceil+2 A_{\max }\right)$, where $\nu_{\max }=\max _{i} \nu_{i}$, the delay of any successfully transmitted packet can be deterministically bounded by $D$ slots.

The loss in utility can be shown to be $\mathcal{O}(1 / D)$ following the proof in [17, Theorem 1] using the following observations: (1) the proposed policy minimises the bound on drift-minus-utility as required, and (2) the virtual queue is also deterministically bounded.

\section{CONCLUDING REMARKS}

We addressed the problem of maximising a concave utility function of long term throughput, subject to deterministic delay guarantees on each dellivered packet. Extending prior work, we allow for any bounded burst distributions for packet arrivals as well as service processes at each link. Introducing non-binary arrival and service processes made the problem considerably harder, and necessitated more careful bounding of the Lyapunov drift.

\section{ACKNOWLEDGEMENTS}

This work was supported in part by the Department of Science and Technology, Government of India. The second author gratefully acknowledges the IU-ATC project, jointly funded by the Department of Science and Technology, Government of India, and the UK EPSRC Digital Economy programme.

\section{REFERENCES}

[1] L. Tassiulas and A. Ephremides, "Dynamic server allocation to parallel queues with randomly varying connectivity," Information Theory, IEEE Transactions on, vol. 39, no. 2, pp. 466 -478, mar 1993.

[2] _ "Stability properties of constrained queueing systems and scheduling policies for maximum throughput in multihop radio networks," Automatic Control, IEEE Transactions on, vol. 37, no. 12, pp. 1936 -1948 , dec 1992.

[3] N. McKeown, A. Mekkittikul, V. Anantharam, and J. Walrand, "Achieving $100 \%$ throughput in an input-queued switch," IEEE Transactions on Communications, vol. 47, no. 8, pp. 1260-1267, 1999.

[4] M. Neely, E. Modiano, and C. Rohrs, "Power and server allocation in a multi-beam satellite with time varying channels," in IEEE INFOCOM, vol. 3, 2002.

[5] — - "Dynamic power allocation and routing for time varying wireless networks," in IEEE INFOCOM, vol. 1, 2003.

[6] A. Brzezinski and E. Modiano, "Dynamic reconfiguration and routing algorithms for IP-over-WDM networks with stochastic traffic,' Journal of Lightwave Technology, vol. 23, no. 10, p. 3188, 2005.

[7] L. Georgiadis, M. Neely, and L. Tassiulas, Resource allocation and cross-layer control in wireless networks. Now Pub, 2006.

[8] X. Lin and N. B. Shroff, "Joint rate control and scheduling in multihop wireless networks," in Decision and Control, 2004. CDC. 43rd IEEE Conference on, vol. 2. IEEE, 2004, pp. 1484-1489. 
[9] A. Eryilmaz and R. Srikant, "Fair resource allocation in wireless networks using queue-length-based scheduling and congestion control," in INFOCOM 2005. 24th Annual Joint Conference of the IEEE Computer and Communications Societies. Proceedings IEEE, vol. 3. IEEE, 2005, pp. 1794-1803.

[10] R. Gallager, Discrete stochastic processes. Kluwer Academic Publishers Boston, 1996, vol. 101

[11] S. Shakkottai and R. Srikant, "Scheduling real-time traffic with deadlines over a wireless channel," Wireless Networks, vol. 8, no. 1, pp. 13-26, 2002.

[12] J. J. Jaramillo and R. Srikant, "Optimal scheduling for fair resource allocation in ad hoc networks with elastic and inelastic traffic," in INFOCOM, 2010 Proceedings IEEE. IEEE, 2010, pp. 1-9.

[13] J. J. Jaramillo, R. Srikant, and L. Ying, "Scheduling for optimal rate allocation in ad hoc networks with heterogeneous delay constraints," Selected Areas in Communications, IEEE Journal on, vol. 29, no. 5, pp 979-987, 2011.

[14] R. Li and A. Eryilmaz, "Scheduling for end-to-end deadline-constrained traffic with reliability requirements in multihop networks," IEEE/ACM Transactions on Networking (TON), vol. 20, no. 5, pp. 1649-1662, 2012

[15] I.-H. Hou, P. Kumar et al., "Admission control and scheduling for qos guarantees for variable-bit-rate applications on wireless channels," in Proceedings of the tenth ACM international symposium on Mobile ad hoc networking and computing. ACM, 2009, pp. 175-184.

[16] I. Hou, V. Borkar, and P. Kumar, "A theory of qos for wireless," INFOCOM 2009, IEEE, pp. 486-494, 2009.

[17] M. J. Neely, "Delay-based network utility maximization," in INFOCOM, 2010 Proceedings IEEE. IEEE, 2010, pp. 1-9.

[18] A. Mekkittikul and N. McKeown, "A practical scheduling algorithm to achieve 100\% throughput in input-queued switches," in INFOCOM'98. Seventeenth Annual Joint Conference of the IEEE Computer and Communications Societies. Proceedings. IEEE, vol. 2. IEEE, pp. 792-799. 